\title{
Molecular Interactions of Carcinogenic Aromatic Amines, 4-Aminobiphenyl and 4,4'-Diaminobiphenyl, with Lactoperoxidase - Insight to Breast Cancer
}

\author{
ISHFAQ AHMAD SHEIKH, MOHD AMIN BEG and MUHAMMAD YASIR
}

\author{
King Fahd Medical Research Center, King Abdulaziz University, Jeddah, Kingdom of Saudi Arabia
}

\begin{abstract}
Background/Aim: Lactoperoxidase (LPO) is an antimicrobial protein present in milk, saliva, gastric secretions, tears and upper respiratory tract secretions. LPO constitutes an important enzyme of the human immune defense system. However, LPO has also been suggested to be involved in breast cancer etiology through production of reactive free radicals and activation of carcinogenic aromatic compounds. Aromatic compounds are generally highly lipophilic and thus accumulate in highly fatty breast tissues. The aromatic compounds 4aminobiphenyl $(A B P)$ and 4,4'-diaminobiphenyl (BZ) are known to have carcinogenic properties. LPO catalyzes their oxidation and converts them into reactive products which bind to DNA and form adducts. These DNA adducts subsequently lead to breast cancer. Materials and methods: The crystal structure of LPO was obtained from Protein Data Bank. Structures of $A B P$ and $B Z$ were retrieved from PubChem database. Induced Fit Docking was performed using glide module from Schrodinger. Results: The present study reports the structural binding of $A B P$ and $B Z$ with $L P O$ using in silico approaches. The amino acid residues of LPO involved in the binding with the two aromatic ligands were characterized and binding energy values were calculated. Conclusion: Both ABP and $B Z$ were placed in the substrate binding site present in the distal heme cavity of LPO with good affinity. The binding mode mimicked that of the natural substrate since these compounds did not disturb the water molecule that plays an important role in the oxidation reaction. Thus, the water molecule is potentially available for facilitating the subsequent activation of the aromatic amines to reactive species which may form DNA adducts leading to breast cancer.
\end{abstract}

Correspondence to: Ishfaq Ahmad Sheikh, King Fahd Medical Research Center, King Abdulaziz University, P.O Box-80216, Jeddah-21589, Kingdom of Saudi Arabia. Tel: +966 532972254, e-mail: iasheikh@kau.edu.sa

Key Words: Breast cancer, docking, lactoperoxidase, 4-aminobiphenyl, 4,4'-diaminobiphenyl.
Breast cancer continues to pose tremendous challenges to the global health. Worldwide, combined for men and women, breast cancer prevalence is second only to lung cancer and is fifth in causing cancer-related deaths (1-4). However, for women, breast cancer is the most common form of cancer constituting about $25 \%$ of all cancers. With about 1.8 million new breast cancer cases in 2013, the number is expected to increase to more than 2 million new cases by the year 2030 $(3,4)$. According to American Cancer Society (2), breast cancer is the second highest cause of cancer deaths among women in the United States. Many factors such as heredity, exposure to estrogen, exposure to environmental carcinogens, dietary carcinogens, etc. have been reported to be associated with breast cancer $(5,6)$. Environmental carcinogens constitute many diverse groups of natural and synthetic chemical compounds including carcinogenic aromatic amines. Carcinogenic aromatic amines were shown to act as mammary carcinogens in rats or mice (7). In the body, aromatic amines undergo metabolic activation forming reactive mutagenic species. An in vitro study on normal human mammary epithelial cells has also shown that that aromatic amines are activated in response to genotoxic products and induce mammary gland cancers (8).

Naturally acting peroxidases in the body mainly lactoperoxidase (LPO) have been shown to activate aromatic amines in breast ducts resulting into mutagenic products (9). LPO is a glycoprotein of around $80 \mathrm{kDa}$ containing calcium and iron (10-12). It is a green color heme-protein present in exocrine gland secretions such as, milk and exhibits antimicrobial and antifungal activities (13-15). Thiocyanate (SCN), present naturally in the human and animal systems, acts as the most preferred substrate for LPO and is oxidized to OSCN, which subsequently acts as an oxidizing agent for bacteria (16). Besides antimicrobial properties, LPO has a role in breast carcinogenesis through activation of carcinogenic aromatic amines such as, benzidine, 2aminofluorene and others, resulting in the formation of metabolites which are highly reactive and bind to DNA covalently (17). Human LPO has also been reported to 


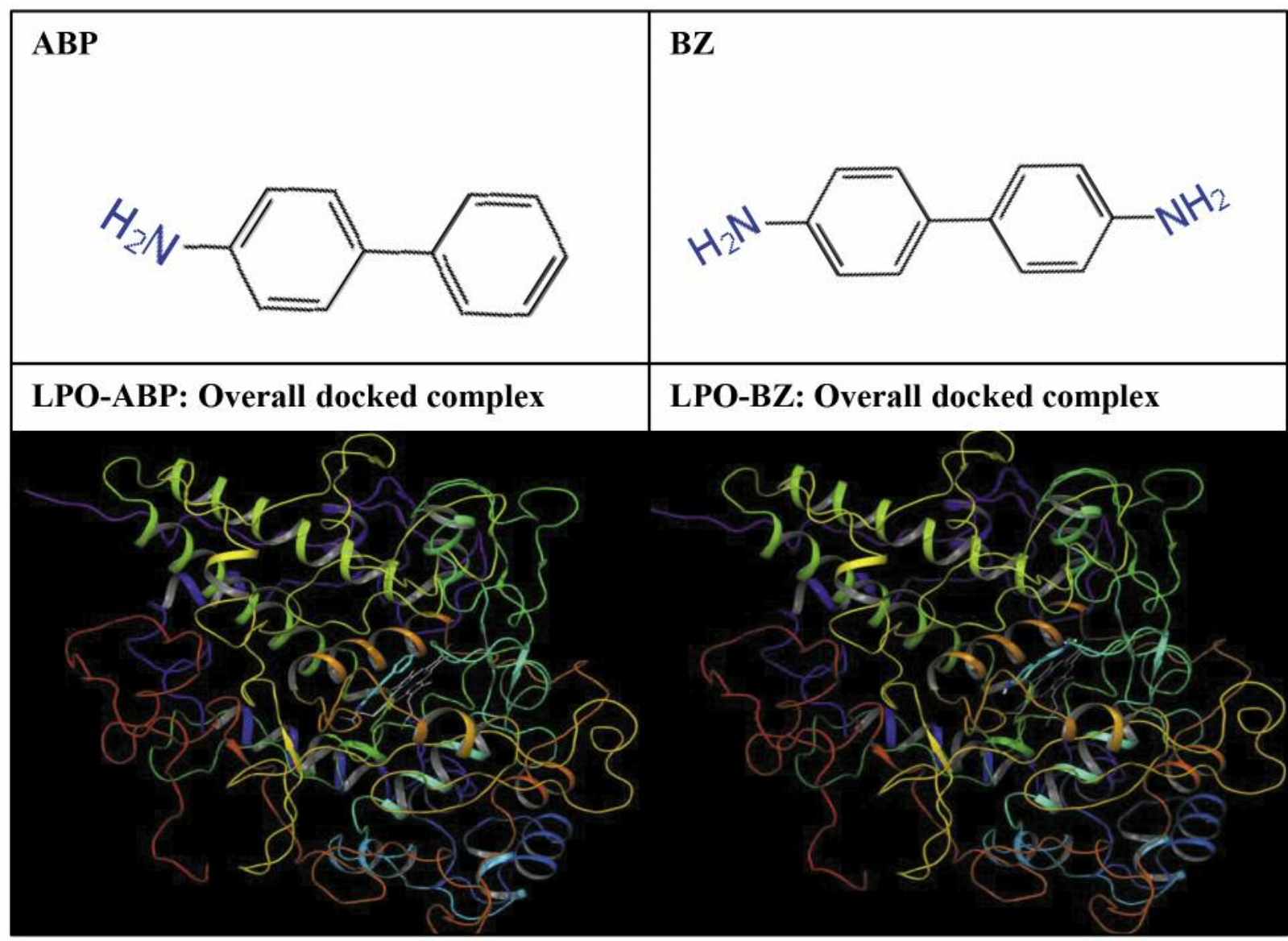

Figure 1. Two dimensional representation of the two aromatic amines, 4-aminobiphenyl (ABP) and 4,4'-diaminobiphenyl (BZ) and ribbon form representation of their docked complexes with lactopeoxidase (LPO).

activate estrogen into stable quinine methides which bind to DNA and initiate breast cancer (18).

Many studies have suggested the activation of aromatic amines by LPO (19-20), but the mechanism of interaction of 4-aminobiphenyl (ABP) and 4,4'-diaminobiphenyl (BZ) with LPO has not been characterized. The current study was performed to provide insights into the structural binding characteristics and the binding mode of these two known carcinogenic aromatic amines, ABP and BZ, with LPO.

\section{Materials and Methods}

The in silico docking simulation studies of LPO with aromatic amines such as ABP and BZ was performed using the Schrodinger 2015 suite (Schrodinger, LLC, New York, USA) as previously described in detail (21).

Data retrieval. The molecular structures of $\mathrm{ABP}$ and $\mathrm{BZ}$ were obtained from the PubChem compound database (https:// pubchem.ncbi.nlm.nih.gov/). Their two dimensional structures are shown in Figure 1 and their PubChem compound identities (CIDs) along with abbreviations are presented in Table I.

Protein selection and preparation. The three dimensional structure of goat LPO complex with 3-hydroxymethyl phenol was retrieved from the Protein Data Bank (PDB) (http://www.rcsb.org) and has a resolution of $1.98 \AA$ (PDB code: $4 \mathrm{MSF}$ ). Human LPO has a very high amino acid sequence identity (over 82\%) with goat LPO, therefore, PDB of the goat LPO complex was used for docking studies. This PDB complex structure was developed using preparation wizard workflow of Schrodinger has been described in detail previously (21).

Ligand preparation. Maestro 10.3 was used for drawing ligand structures (Maestro, version 10.3; Schrodinger). For further analysis, ligands were prepared using LigPrep module (LigPrep, version 3.1; Schrodinger). The methodology has been described previously (21).

Induced fit docking. Prime module of Schrodinger 2015 suite was used for the execution of induced fit docking. 


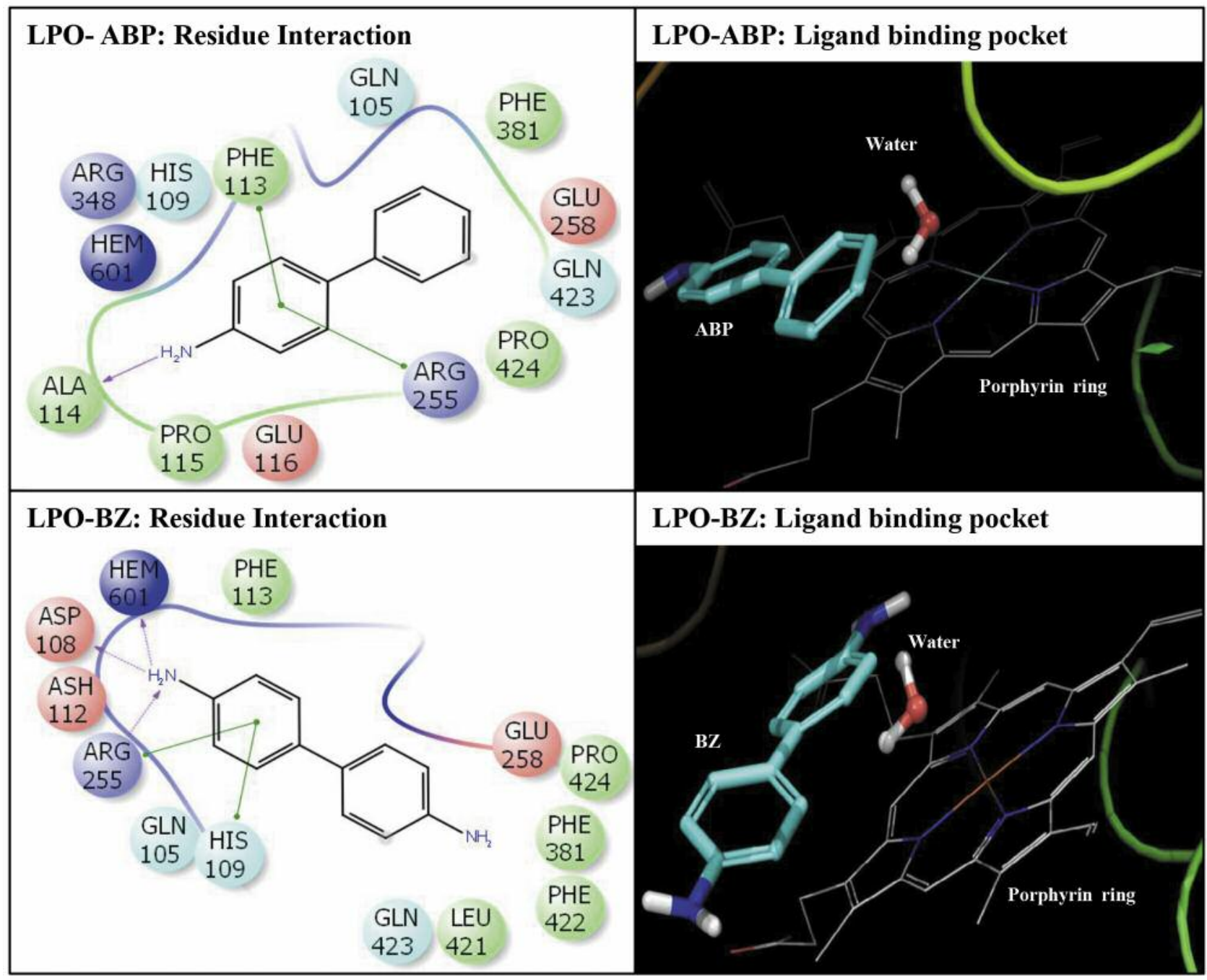

Figure 2. Amino-acid residue interaction of two aromatic amines, 4-aminobiphenyl (ABP) and 4,4'-diaminobiphenyl (BZ) in the binding pocket of lactopeorxidae (LPO) and their binding pattern in distal heme cavity.

Table I. Nomenclature, commonly used abbreviations, and PubChem IDs of the two aromatic compounds.

\begin{tabular}{lccc}
\hline S.No. & Name & Abbreviation & PubChem ID \\
\hline 1 & 4-Aminobiphenyl & ABP & 7102 \\
2 & 4,4'-Diaminobiphenyl & BZ & 7111 \\
\hline
\end{tabular}

Binding energy calculations. For calculating the binding affinity of ABP and BZ with LPO, MM-GBSA (Molecular Mechanics with Generalized Born and Surface Area) model present in Prime module of Schrodinger 2015-3 was used.

\section{Results}

Molecular docking. The amino acid residues of LPO interacting with $\mathrm{ABP}$ and $\mathrm{BZ}$ are shown in Figure 2. In the LPO-ABP complexes, 13 residues of LPO were engaged in interactions with $\mathrm{ABP}$, whereas in LPO-BZ complex, 12 residues of $\mathrm{LPO}$ were involved in interactions with $\mathrm{BZ}$. Additionally, both ABP and BZ exhibited interactions with heme ring. Eight interacting residues (Gln-105, His-109, Phe-113, Arg-255, Glu-258, Phe-381, Gln-423, Pro-424) were common in both the complexes. In addition, both ABP and BZ exhibited a common pi-pi interaction with Arg-255. Further, ABP displayed a pi-pi interactions with Phe-113, whereas, BZ displayed pi-pi interactions with His-109. 
Table II. Number of interacting residues, Dock score, Glide score and binding affinity values (MMGB-SA values) 4-aminobiphenyl (ABP) and 4,4'diaminobiphenyl (BZ) after IFD with lactoperoxidase (LPO).

\begin{tabular}{lccccc}
\hline Target & Ligand & Number of interacting residues & Docking score (Kcal/mol) & Glide score (Kcal/mol) & MMGB-SA (Kcal/mol) \\
\hline LPO & ABP & 13 & -6.16 & -6.16 & -51.81 \\
LPO & BZ & 12 & -6.34 & -6.36 & -49.51 \\
\hline
\end{tabular}

Moreover, one hydrogen bonding interaction between ABP and Ala-114, and three hydrogen bonding interactions between BZ and Asp-108, Arg-255 and the heme ring were also observed. Both ligands bound in the distal heme cavity of LPO at the substrate binding site, but did not disturb the important water molecule required for the oxidation reaction to occur (Figure 2). Binding affinity values, glide score, and dock score for both the ligands have also been calculated and are presented in Table II.

\section{Discussion}

Both natural and man-made aromatic amines exist in our environment and have been associated with cancer (22-25). Several aromatic amines, such as 3,3'-dimethoxybenzidine (26), ABP, 2-aminofluorene (27), and 2-amino-l-methyl-6phenylimidazo[4,56]pyridine (28) have been reported to induce mammary tumors in female rats. An exhaustive review on scientific investigations carried under the supervision of the National Toxicology Program, USA, on rodent mammary carcinogens indicated that 16 of 34 carcinogens were aromatic amines or nitro compounds (7). It has been shown that activation of ABP by LPO in the presence of hydrogen peroxide $\left(\mathrm{H}_{2} \mathrm{O}_{2}\right)$ resulted in four different carcinogenic reaction products after 15 minutes, whereas similar activation of BZ resulted in one reaction product (20). Additionally, susceptibility to enzymatic activation was highest for BZ followed by ABP, and BZ also displayed highest level of binding to DNA. Further, it was shown that DNA adducts were formed in breast duct epithelial cells of women exposed to aromatic amines (19).

In the current study, both ABP and BZ were shown to bind to LPO in the distal heme cavity region without disturbing the critical water molecule. Previously (16), this water molecule present in the distal heme cavity of LPO was shown to be crucial for the oxidation reaction. One of the important characteristics which distinguish inhibitors from substrates of LPO is the fate of this crucial water molecule upon ligand binding in the distal heme cavity. Inhibitors, upon binding to LPO, disturb this water molecule and subsequently do not allow oxidation reaction but in case of substrates, this water molecule in the distal heme cavity is not perturbed (29). As observed in the present study, this water molecule is available for oxidation of aromatic amines $\mathrm{ABP}$ and BZ bound in the distal heme cavity of LPO, therefore, are activated to highly reactive species that bind to DNA. Binding of these reactive products has been suggested to lead to alterations in the genetic material of milk ducts which has been suggested to be one of the causes of cancer in the mammary tissue (20).

In conclusion, this study showed that aromatic amines ABP and BZ bind in the distal heme cavity of lactoperoxidase with great affinity and their mode of binding is similar to that of LPO substrates as the crucial water molecule essential for oxidation reaction was not disturbed. Thus, the water molecule is potentially available for facilitating the subsequent activation of the aromatic amines to reactive species which may form DNA adducts leading to breast cancer.

\section{Conflicts of Interest}

The Authors have declared that no competing interests exist.

\section{Acknowledgements}

This project was funded by the Deanship of Scientific Research (DSR) at King Abdulaziz University, Jeddah, under grant no. (G323/141/1434). The Authors, therefore, acknowledge with thanks DSR for its technical and financial support.

\section{References}

1 Ferlay J, Soerjomataram I, Dikshit R, Eser S, Mathers C, Rebelo M, Parkin DM, Forman D and Bray F: Cancer incidence and mortality worldwide: sources, methods and major patterns in GLOBOCAN 2012. Int J Cancer 136(5): E359-386, 2015.

2 ACS. Global cancer facts \& figures, 3rd edition, Atlanta. Atlanta: American Cancer Society; 2015. (https:// www.cancer.org/content/dam/cancer-org/research/ cancer-factsand-statistics/global-cancer-facts-and-figures/global-cancer-factsand-figures-3rd-edition.pdf).

3 Jemal A, Bray F, Melissa MC, Jacques F, Elizabeth W and Forman D: Global cancer statistics. CA Cancer J Clin 61: 69-90, 2011.

4 Global Burden of Disease Cancer Collaboration: The global burden of cancer 2013. JAMA Oncol 1(4): 505-527, 2015.

5 Harris JR, Lippman MW, Veronesi U and Willet W: Breast cancer (1). N Engl J Med 327: 319-328, 1992. 
6 Henderson IC: Risk factors for breast cancer development Cancer 71: 2127-2140, 1993.

7 Dunnick JK, Elwell MR, Huff J and Barrett JC: Chemically induced mammary gland cancer in the National Toxicology Program's carcinogenesis bioassay. Carcinogenesis 16: 173-179, 1995.

8 Eldridge SR, Gould MN and Butterworth BE: Genotoxicity of environmental agents in human mammary epithelial cells Cancer Res 52: 5617-5621, 1992.

9 Josephy PD: The role of peroxidase-catalyzed activation of aromatic amines in breast cancer. Mutagenesis 11(1): 3-7, 1996.

10 Furtmuller PG, Jantschko W, Regelsberger G, Jakopitsch C, Arnhold $\mathrm{J}$ and Obinger $\mathrm{C}$ : Reaction of lactoperoxidase compound I with halides and thiocyanate. Biochemistry 41(39): 11895-11900, 2002.

11 Davies MJ, Hawkins CL, Pattison DI and Rees MD: Mammalian heme peroxidases: from molecular mechanisms to health implications. Antioxidants and Redox Signaling 10(7): 1199-1234, 2008

12 deWit JN and van Hooydonk ACM: Structure, functions and applications of lactoperoxidase in natural antimicrobial systems. Nederlands melk en Zuiveltijdschrift 50(2): 227-244, 1996.

13 Ahariz $M$ and Courtois P: Candida albicans susceptibility to lactoperoxidase-generated hypoiodite. Clin Cosmet and Investig Dent 2: 69-78, 2010.

14 Welk A, Meller C, Schubert R, Schwahn C, Kramer A and Below $\mathrm{H}$ : Effect of lactoperoxidase on the antimicrobial effectiveness of the thiocyanate hydrogen peroxide combination in a quantitative suspension test. BMC Microbiology 9(134): 1-8, 2009.

15 Pruitt KM and Tenovou JO: The Lactoperoxidase System. Chemistry and biological significance. Marcel Dekker (eds.), New York, USA, pp. 1-257, 1985.

16 Sheikh IA, Singh AK, SinghN, Sinha M, Singh SB, Bhushan A, Kaur P, Srinivasan A, Sharma S and Singh TP: Structural evidence of substrate specificity in mammalian peroxidases: Structure of the thiocyanate complex with lactoperoxidase and its interactions at $2.4 \AA$ A resolution. J Biol Chem 284(22): 14849 1456, 2009

17 Malejka-Giganti D and Ritter CL: Peroxidative metabolism of carcinogenic $\mathrm{N}$-arylhydroxamic acids: implications for tumorigenesis. Environ Hlth Perspect 102(Suppl 6): 75-81, 1994.

18 Gaikwad NW: Metabolomic profiling unravels DNA adducts in human breast that are formed from peroxidase mediated activation of estrogens to quinone methides. PLoS One 8(6): e65826, 2013

19 Gorlewska-Roberts KM, Green B, Fares M, Ambrosone CB and Kadlubar FF: Carcinogen-DNA adducts in human breast epithelial cells. Environ Mol Mutagen 39: 184-192, 2002.
20 Gorlewska-Roberts KM, Teitel CH, Lay JO Jr, Roberts DW and Kadlubar FF: Lactoperoxidase-catalyzed activation of carcinogenic aromatic and heterocyclic amines. Chem Res Toxicol 17(12): 1659-1666, 2004.

21 Sheikh IA: Stereoselectivity and potential endocrine disrupting activity of Bis-(2-ethylhexyl)phthalate (DEHP) against human progesterone receptor: a computational perspective. J Appl Toxicol 36(5): 741-747, 2016.

22 Ezaki H, Ambrosone CB, Freudenheim JL, Graham S, Marshall JR, Vena JE, Brasure JR and Michalek AM: Cigarette smoking, $\mathrm{N}$-acetyltransferase 2 genetic polymorphisms and breast cancer risk. J Am Med Assoc 276: 1494-1501, 1996.

23 DeBruin LS, Pawliszyn JB, and Josephy PD: Detection of monocyclic andaromatic amines, possible mammary carcinogens, in human milk. Chem Res Toxicol 12: 78-82, 1999.

24 Zheng W, Gustafson DR, Sinha R, Cerhan JR, Moore D, Hong $\mathrm{CP}$ and Anderson KE: Well-done meat intake and the risk of breast cancer. J Natl Cancer Inst 90: 1724-1729, 1998.

25 Williams JA, and Phillips DH: Mammary expression of xenobiotic metabolizing enzymes and their potential role in breast cancer. Cancer Res 60: 4667-4677, 2000.

26 Morgan DL, Dunnick JK, Goehl T, Jokinen MP, Matthews HB, Zeiger E and Mennear JH: Summary of the National Toxicology Program benzidine dye initiative. Environ Hlth Perspect 102(Suppl 2): 63-78, 1994.

27 Garner RC, Martin CN and Clayson DB: Carcinogenic aromatic amines and related compounds. In: Chemical Carcinogens. Searle CE (ed.), 2nd edn. American Chemical Society, Washington, DC, pp. 175-276, 1984.

28 Ito N, Hasegawa R, Sano M, Tamano S, Esumi H, Takayama S and Sugimura T: A new colon and mammary carcinogen in cooked food, 2-amino-1-methyl-6-phenyhmidazo[ $4^{\wedge}$-fc]pyridine (PhIP). Carcinogenesis 12: 1503-1506, 1991.

29 Singh AK, Singh N, Sinha M, Bhushan A, Kaur P, Srinivasan A, Sharma S and Singh TP: Binding modes of aromatic ligands to mammalian heme peroxidases with associated functional implications: crystal structures of lactoperoxidase complexes with acetylsalicylic acid, salicylhydroxamic acid, and benzylhydroxamic acid. J Biol Chem 284(30): 20311-20318, 2009.
Received March 27, 2017

Revised April 10, 2017

Accepted April 12, 2017 